\title{
REPLICAÇÃO DE FÓSSEIS DE FORAMINIFERA UTILIZANDO REAPROVEITAMENTO DE “ISOPOR" COM ALUNOS DO IFPB-CG
}

\author{
H. O. Machado-Filho, A. C. M. Ferreira e D. R. Soares \\ Instituto Federal da Paraíba - Campus Campina Grande (IFPB-CG) \\ hermes@ifpb.edu.br - a.claudia1@hotmail.com - dwight@ifpb.edu.br
}

Artigo submetido em março/2011 e aceito em agosto/2011

\section{RESUMO}

Os fósseis são unidades paleontológicas preciosas e difíceis de serem coletadas para pesquisas ou principalmente para aulas práticas sobre o passado geológico da Terra. Desta forma, buscamos técnicas alternativas para se fazer réplicas dessas unidades. 0 presente artigo vem apresentar atividades de sistemática paleozoológica, utilizando o "isopor" como matéria prima para confecção de modelos de fósseis em aulas práticas para a comunidade escolar do Instituto Federal de Educação, Ciência e Tecnologia da Paraíba, campus de Campina Grande. Dessa forma, o instituto está iniciando a construção de um acervo visual 3D alternativo de fósseis guia do grupo dos Foraminifera, em específico, os bioindicadores de petróleo, além de contribuir para reutilização de resíduos sólidos poluidores ao meio ambiente.

PALAVRAS-CHAVE: Paleontologia; Resíduos Sólidos; Acervo IFPB.

\section{FOSSIL FORAMINIFERA REPLICATION USING REUSE OF "STYROFOAM" WITH STUDENTS FROM IFPB-CG}

\section{ABSTRACT}

The units paleontological fossils are precious and difficult to be collected primarily for research or for practical classes on the Earth's geological past. Thus, we seek alternative techniques to make replicas of these units. This article come from to present systematic paleozoology activities, using "foam" as raw material to making models of fossils in practical classes for the school community at the Instituto Federal de Educação, Ciência e Tecnologia da Paraíba, campus Campina Grande. Thus, the institute is beginning to build a decent 3D visual guide alternative fossil Foraminifera of the group, in particular, the biomarkers of petroleum, and contribute to reuse of solid waste polluting the environment.

KEY-WORDS: Paleontology; Solid Waste, Collection IFPB. 


\section{REPLICAÇÃO DE FÓSSEIS DE FORAMINIFERA UTILIZANDO REAPROVEITAMENTO DE “ISOPOR" COM ALUNOS DO IFPB-CG}

\section{INTRODUÇÃO}

A Paleontologia é uma ciência que estuda as evidências da vida, a partir da pesquisa com fósseis de animais, plantas, microorganismos; seres que viveram no nosso planeta em determinados períodos da história natural, e que foram confinados dentro das rochas sedimentares, por algum evento do passado geológico da Terra (CARVALHO, 2004).

Em vários casos, o estudo da Paleontologia fica comprometido pela escassez de material fossilífero, pois essas formações rochosas são raras, protegidas por lei ( $\left.N^{\circ} 4.146 / 42\right)$, e muitas vezes não podem ser coletadas amostras, dadas as limitações da condição humana (dificuldade de acesso a determinadas áreas, falta de equipamento especializado, mão de obra qualificada, etc).

Uma forma simples de se estudar esse passado geológico, utilizando conhecimentos paleontológicos, é através da confecção de fósseis por diversos métodos de modelagem e replicação, seja por cerâmica, gesso, madeira, vidro (SAMPAIO, et al, 2002), assim como também, pelo material conhecido por "isopor", que é uma substância inerte, amorfa e maciça de uso comercial.

Geralmente, após a utilização imediata do isopor, principalmente para embalagem de eletrodomésticos ou objetos quebradiços, ele é descartado gerando um acúmulo de resíduo que além de não apresentar utilidade ao homem, poluí o meio ambiente.

Conhecida a problemática ambiental atual, onde há uma crescente produção de lixo, fato este que vem poluindo a natureza e o espaço humanizado, e sabendo que o "isopor" é mais um desses agentes que corrobora para aumentar a poluição do meio ambiente, por não ser biodegradável e demorar milhões de anos para se decompor na natureza (JERÔNIMO, 2004), esse material precisa ser reaproveitado pela cadeia produtiva humana de forma a minimizar os seus efeitos poluentes.

Dessa forma, surge à iniciativa do presente trabalho, em se criar um acervo paleontológico alternativo para uso na comunidade do Instituto Federal de Educação, Ciência e Tecnologia da Paraíba, campus Campina Grande (IFPB-CG). O Instituto oferece cursos de na área de Mineração, entre outros, e os alunos precisam ter práticas de paleontologia para prospecção e identificação de fósseis bioindicadores de bens minerais. Nesta primeira etapa organizaram-se alguns fósseis guia do grupo dos Foraminifera, de algumas espécies da costa atlântica brasileira.

\section{MATERIAL E MÉTODOS}

A metodologia se baseou primeiramente na organização ontológica do estudo sobre foraminíferos e, posteriormente, a parte prática da confecção de modelos. Ontologia é um modelo de dados que representa um conjunto de conceitos dentro de um domínio e os relacionamentos entre estes (GUELERI, et al, 2008). A premissa ontológica é utilizada para realizar estudos aprofundados e inferências sobre os objetos do domínio. 
Os procedimentos metodológicos são de toda forma bem simples e vão se basear, principalmente, nas obras realizadas por Anelli, et al (2004), Duleba, et al (2007), Simone (1994), Tinoco (1959). Anelli, et al (2004), que descrevem com exatidão a importância, métodos e técnicas de se confeccionar modelos de fósseis de forma criativa e de baixo custo; Simone (1994) traz um apanhado dos principais tipos de fósseis marinhos (moluscos) encontrados no Brasil, Muniz (1993) trata dos fósseis da região da extensão Paraíba-Pernambuco, Duleba, et al (2007) versa sobre um dos principais estudos sobre métodos de controle bioestratigráfico, ou seja, descrições sobre os ambientes de deposição fossilíferos paleoceanográficos, e Tinoco (1959) traz uma coleção sobre fósseis guia de foraminíferos da costa brasileira, especificadamente, a pernambucana.

O local de coleta dos testemunhos foi o litoral do Município de Olinda - PE (Tinoco, 1958) e os isopores foram coletados aleatoriamente pelo comércio do centro do Município de Campina Grande PB.

As famílias destacadas na pesquisa foram: Textularidae, Valvulinidae, Miliolidae, Ophthalmidiidae, Trochamminidae, Nodosariidae, Lagenidae, Nonionidae, Camerinidae, Peneroplidae, Bulminidae, Rotalidae, Amphisteginidae, Cymbaloporidae, Cassidulinidae, Globigerinidae, Globorotalidae, Anomalinidae e Planorbulinidae.

A pesquisa seguiu a seguinte linha de desenvolvimento dos trabalhos:

- Organização de um grupo de estudos sobre Paleontologia com pesquisas em livros técnicos e manuais específicos na tentativa de se estabelecer um resgate bibliográfico sobre o material referente ao tema, para dessa forma, detalhar alguns fósseis de cada período geológico, identificar quais são os mais representativos, quais fósseis os livros registram com maior freqüência, para definir com maior clareza quais modelos de fósseis seriam fabricados a princípio pelo projeto;

- Elaboração de fichamentos, através da pesquisa bibliográfica sobre cada fóssil escolhido/analisado, morfologia específica, o possível ramo evolutivo da espécie, características relacionadas com a paleoecologia, paleoclimatologia e paleogeografia da referida entidade paleontológica;

- Coleta de isopor em estabelecimentos comerciais do centro da cidade de Campina Grande, no fim do expediente, finais de tarde, pois nesse horário geralmente as empresas costumam colocam seus resíduos para serem coletados pelo serviço de limpeza urbana e catadores de lixo;

- Confecção dos modelos bidimensionais ou tridimensionais dos fósseis a partir dos procedimentos de técnica de escultura em poliestireno expandido. 
O espaço de realização das atividades foi o Laboratório de Química do Instituto Federal de Educação, Ciência e Tecnologia, campus Campina Grande (IFPB-CG). O público alvo que está sendo beneficiado são os alunos do grupo de estudos e, posteriormente, os do curso de Mineração do Instituto, que aos poucos, também estão se integrando as práticas de escultura dos fósseis.

A técnica para a reutilização do isopor ou poliestireno expandido foi através da visualização direta dos manuais de identificação e escultura em pedaços de isopor através de espátula, estilete e lixa comum.

Os passos para a confecção das peças em poliestireno expandido são os seguintes:

- O fóssil guia ou a figura que serve como modelo é colocado em local livre e iluminado, onde fica até o término das atividades;

- Delimita-se no pedaço de poliestireno expandido, com um lápis hidrocor a forma do fóssil que vai ser esculpido e retira as arrestas com estilete, entalhando com uma espátula, dando a forma desejada ao objeto;

- Caso a peça precise de um contorno mais aguçado, pode-se esquentar a lâmina da espátula com um bico de Bunsei e formatar melhor o artefato.

\section{RESULTADOS}

\section{Caracterização Geral no Estudo de Foraminifera}

Os Foraminifera ou foraminíferos são proctistas bentônicos ou planctônicos que surgiram no Período Cambriano, portanto há cerca de 570 milhões de anos, e vivem até o Recente (MENDES, 1982). São seres carapaçados ou que possuem uma teca de forma variada, composta de carbonato de cálcio e/ou aglutinada por partículas do sedimento em que vive ou, mais raramente, orgânica ou silicosa (op. cit).

A classificação desse grupo constitui-se por diferenças morfológicas, o hábito ambiental e ocorrência no tempo geológico (DULEBA, et al, 2004). Desta forma, é possível retirar várias correlações sobre as espécies de foraminíferos, pois esses animais constituem testemunhos únicos sobre as variações no passado da terra, no campo biológico, ecológico e geográfico.

A variedade de formas e estruturas de teca e carapaças em geral e de seus elementos formadores torna a classificação dos foraminíferos muito complexa, mas ao mesmo tempo facilita a diferenciação entre os taxa (DULEBA, et al, 2004). Para a caracterização das famílias, gêneros e espécies leva-se em consideração, por exemplo, o número e o formato das câmaras de contato com o ambiente externo, linhas de sutura morfológicas, formato geral e diferentes tipos de ornamentos. 
Os estudos ecológicos sobre a fauna Foraminifera geralmente são executados pela bioestratigrafia de sedimentos rochosos em afloramentos específicos (MENDES, 1982). A partir dessas formações rochosas pode-se ter uma visão generalista sobre a vida no passado da Terra, seu funcionamento e organização, populações e comunidades desses animais dentro dos ecossistemas.

Os registros desses organismos apresentam uma dimensão geográfica no que tange a sua distribuição paleogeográfica e os achados fossilíferos na configuração do globo atual (MENDES, 1982). Através de técnicas de análise sedimentológica do ambiente, podem-se identificar as mudanças no nível e na localização do oceano primitivo, tais como e onde ocorreu a deposição do material que contém as peças.

Desta forma, pelo que se pode perceber, o estudo de foraminíferos é quase totalmente restrito a observações de levantamentos taxonômicos, com utilização de técnicas de campo e de microscopia (DULEBA, et al, 2004).

A pesquisa de campo é um obstáculo para esses estudos. Caso o local seja acessível ou a instituição pesquisadora promova a exploração de uma área, oferecendo, assim, condições para o feitio, então tudo se torna mais fácil. Mas na maioria das vezes os ambientes de exploração são inacessíveis, tais como assoalho de oceano e fundo de geleiras, e quando acessíveis precisam da utilização de equipamentos específicos.

No caso da pesquisa laboratorial, na utilização de microscópio eletrônico, geralmente não provoca um impacto em quem visualiza a lâmina, pois os fósseis na realidade não são tão perfeitos como os que aparecem em muitas estampas desenhadas à mão. É bastante comum na pesquisa científica desses seres a escassez de material visual de qualidade.

Quando se tem acesso a equipamentos de alta tecnologia, como microscópios de alta resolução e precisão, podem-se conseguir bons resultados, porém a aquisição desses equipamentos tem um alto custo.

Observa-se, na figura 1, um conjunto de estampas confeccionadas pela equipe do laboratório de Paleontologia da Universidade de São Paulo, através de microscópios de varredura digital, que também já foram identificados por Tinoco (1958). 

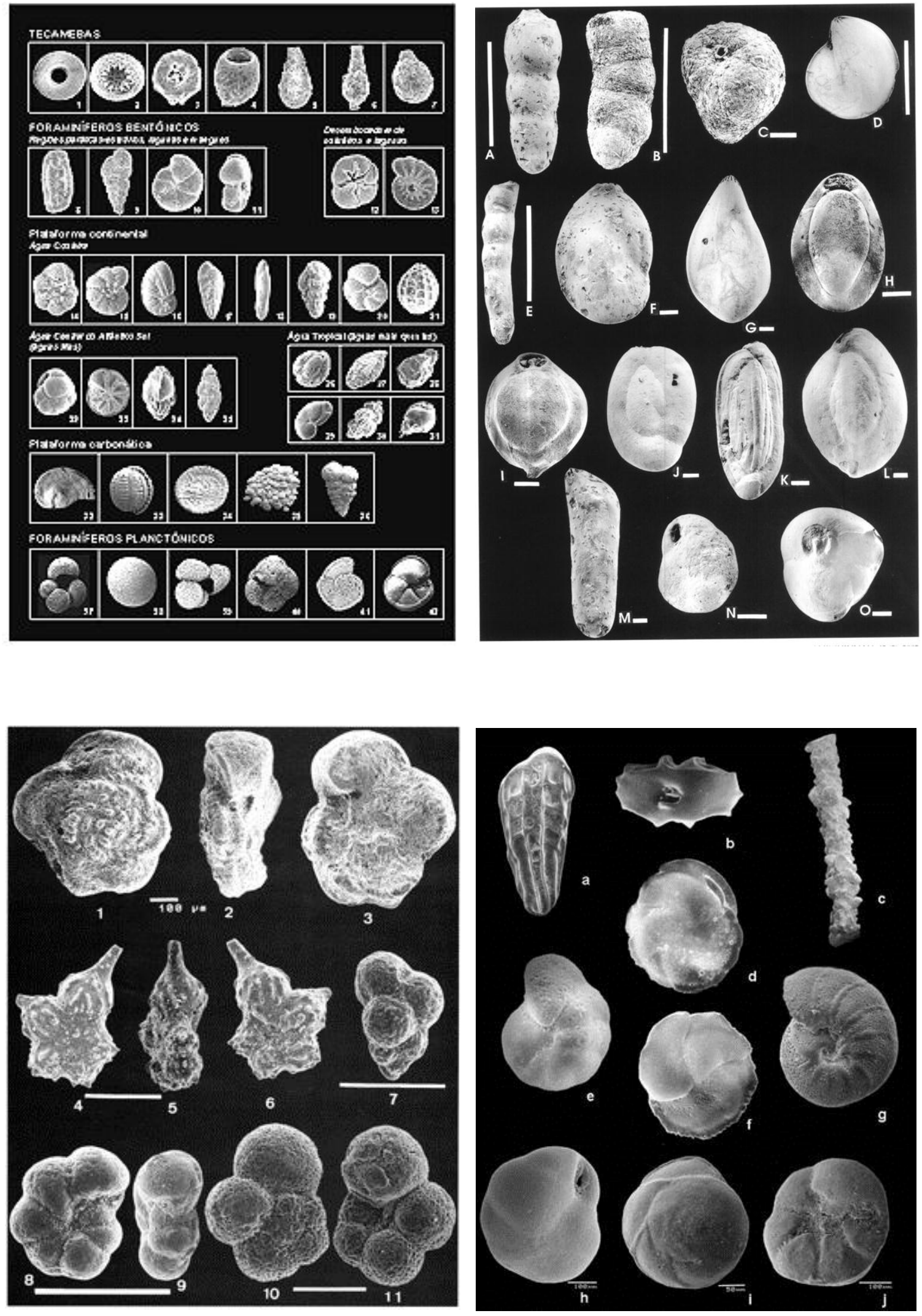

Figura 1: Conjunto de estampas contendo espécies de foraminíferos oceânicos.

HOLOS, Ano 27, Vol 4 
Fonte: Disponível no site www2.igc.usp.br/micropaleo/laboratorio.htm. Acesso em: 23/07/2009.

De posse desse conhecimento, a montagem de material visual (e em escala ampliada) é uma alternativa viável para auxiliar na prospecção de microfósseis de foraminíferos, pois tanto apresenta um cunho didático mais interativo na relação ensino-aprendizagem como na apreensão de determinados conceitos, situações e características específicas para estudos de micropaleontologia.

\section{Reaproveitamento de Isopor para a Pesquisa Científica de Foraminiferos}

O isopor é um tipo de espuma leve e volumosa que apresenta uma vasta utilização na indústria e no comércio. Apresenta-se como um material moldado, constituído por um aglomerado de grânulos sintéticos, a partir de derivados do petróleo (REIS, 2003).

Na natureza o isopor leva 150 anos para ser degradado, quimicamente, conforme estimativas, o isopor consiste apenas de dois elementos naturais, o carbono e o hidrogênio (REIS, 2003). O isopor não contém qualquer produto tóxico ou perigoso para o ambiente e para a camada de ozônio (está isento de (FCs), mas se caracteriza como resíduo sólido que provoca alguns inconvenientes, como sujeiras nas vias públicas, podendo até ser confundido e engolido por animais, causando problemas de saúde.

$\mathrm{O}$ isopor pode ser considerado, desta forma, um produto potencialmente danoso ao ambiente, já que polui o solo e a água. Porém ele é um material 100\% reaproveitável nas cadeias produtivas humanas, quando utilizado, por exemplo, para a prática do artesanato ou em atividades escolares.

Desta forma cabe também o desenvolvimento de pesquisas nessa temática para propor outras formas no seu reaproveitamento e assim contribuir para a diminuição de resíduos sólidos, possibilitando uma melhor qualidade de vida ambiental.

A seguir, observam-se algumas imagens de espécimes já confeccionados na coleção didáticocientífica (figura 2), e outras imagens de espécimes com suas respectivas especificações morfoestruturais para a identificação sistemática e taxonômica da espécie estudada. 


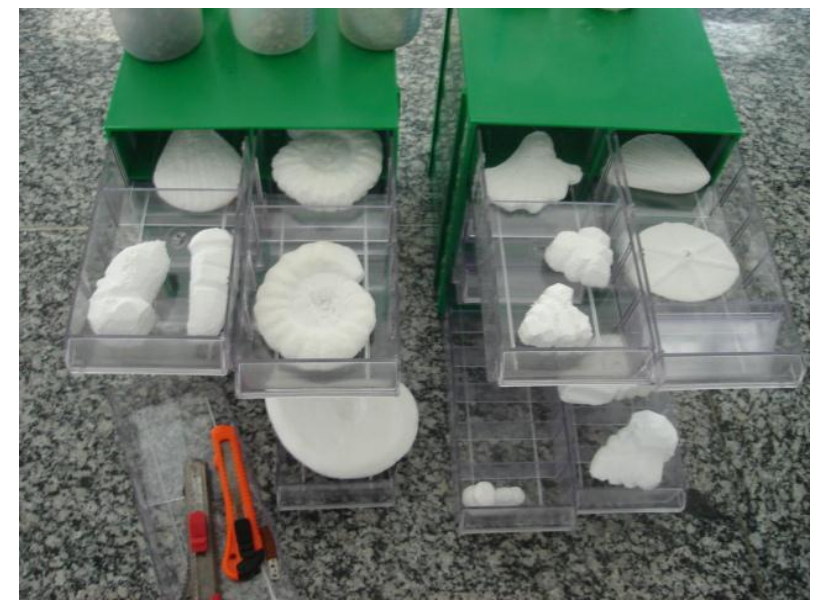

Figura 2: Imagens dos materiais elaborados no IFPB pela reutilização do isopor para confecção de replicas de fosseis.

Fonte: Arquivo dos Autores.

A figura 3 apresenta o protótipo esquemático do foraminífero Textularia agglutinans D’Orbigny-Cushan, 1992 (Tinoco, 1958). Sua morfologia se baseia numa testa alongada, afinando-se gradualmente para uma das extremidades distintas e deprimidas, paredes arenosas de granulação um tanto grosseiras; abertura representada por uma fenda alongada na base de uma depressão na margem interna da última câmara.

As dimensões desse animal são $0,68 \mathrm{~mm}$ e sua largura mede $0,37 \mathrm{~mm}$ na realidade. Para a elaboração de sua réplica, foi aumentado 10x de forma escalar.
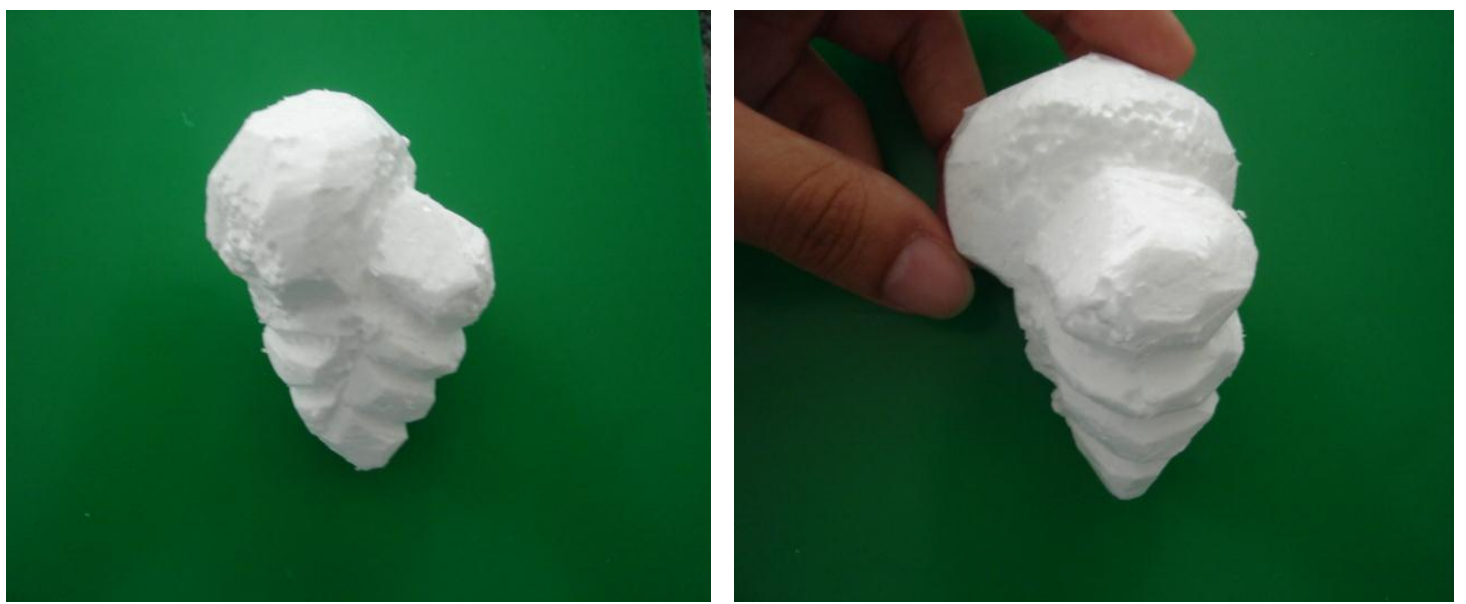

Figura 3: Imagens do foraminífero Textularia agglutinans frotalmente e de perfil, respectivamente. 
A figura 4 demonstra a elaboração de outra espécie do gênero Textularia, o espécime Textularia conica D'Orbigny-Cushan, 1992 (Tinoco, 1958), cujo comprimento é menor que a largura, apresentando um contorno triangular, seção transversal oval, ligeiramente comprimida; câmaras relativamente poucas e distintas, apresentando suturas pouco nítidas, deprimidas, abertura formada por uma fenda na base interna da ultima câmara formada.

A dimensão desse espécime varia em torno de $0,21 \mathrm{~mm}$, largura $0,25 \mathrm{~mm}$, espessura $0,10 \mathrm{~mm}$. E para a elaboração da sua réplica, também foi aumentada 10x o exemplar de forma escalar.

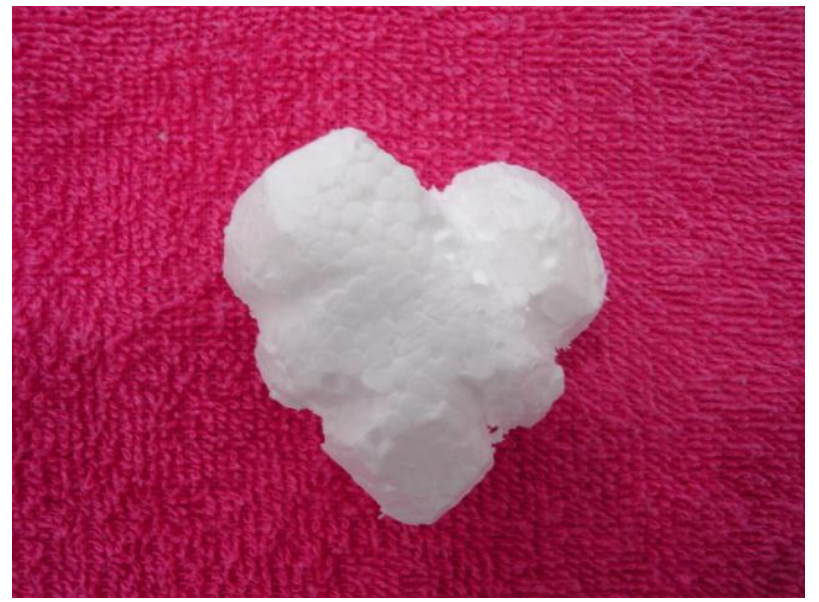

Figura 4: Imagens do foraminífero Textularia conica visto frotalmente.

Em seguida observa-se alguns exemplares pré-finalizados, e lixados na figura 5 e 6.

A figura 5 mostra um espécime de Gibicides pseudoumgeriana Cushman, 1931 (TINOCO, 1958). Apresenta uma testa plana convexa lado dorsal aproximadamente plano, lado ventral convexo; periferia subaguda, câmaras numerosas, todas vistas do lado dorsal, só as da última volta, em numero 10, são observadas ventralmente aumentando gradualmente de tamanho à proporção que são adicionadas; suturas dorsais oblíquas, levemente curvadas, limbadas, principalmente na porção primitiva da testa, deprimidas na porção mais nova; suturas ventrais deprimidas na porção mais nova; paredes grosseiramente perfuradas no lado dorsal; lado ventral finamente perfurado, abertura periférica, interessando pouco o lado dorsal.

Suas dimensões não ultrapassam de $0,55 \mathrm{~mm}$ e foi realizada uma réplica de tamanho $10 x$ maior, em proporção escalar. 



Figura 5: Imagens do foraminífero Gibicides pseudoumgeriana frotalmente e de perfil, respectivamente

Na figura 6, visualiza-se a Anomalina sp. "A" d'Olbigny 1826 (TINOCO, 1958). Trata-se de um foraminífero de testa biconvexa, comprimida e periferia arredondada, câmaras infladas, arranjadas em duas voltas, todas visíveis do lado dorsal, apenas as câmaras da última volta, em numero 8, são observáveis do lado ventral; suturas ligeiramente arqueadas, deprimidas, excerto na volta primitiva, onde são limbadas e niveladas com a superfície, paredes grosseiramente perfuradas labiada, constituída por uma fenda na base da última câmara formada.

A sistemática dessa espécie está ligada a $A$. grosserrugosa Resus. As dimensões dessa Anomalina estudada é de $0,31 \mathrm{~mm}$, espessura de $0,14 \mathrm{~mm}$. Com aumento de $10 x$ para sua réplica.
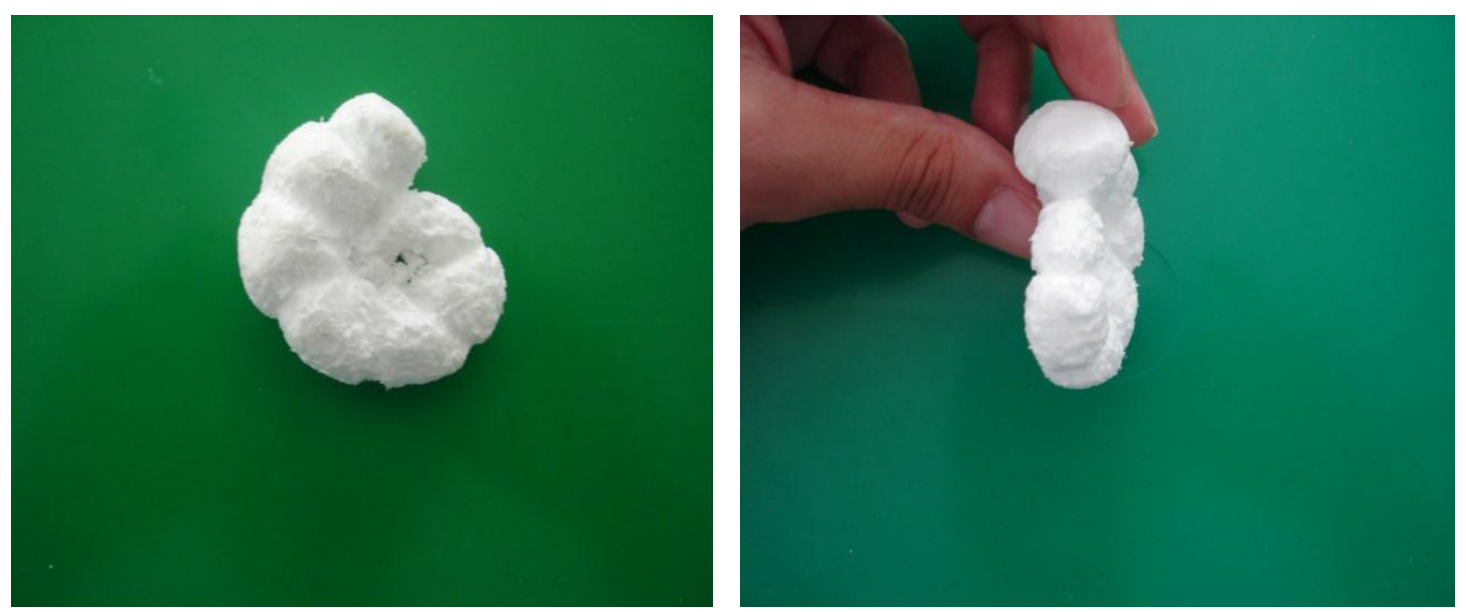

Figura 6: Imagens do foraminífero Anomalina sp. frotalmente e de perfil, respectivamente 


\section{CONSIDERAÇÕES FINAIS}

A Paleontologia é uma ciência antiga e calcada num conhecimento complexo. Seu saber se constrói a partir da pesquisa geológica tradicional, porém pode-se percorrer atualmente a novas metodologias para se fazer gerar informação paleontológica através de inovações criativas interdisciplinares.

O presente trabalho buscou apresentar uma nova metodologia ecologicamente correta em se estudar e analisar espécies paleontológicas guia, para gerar conhecimento sobre a noção de bioindicadores ambientais aplicados à prospecção de petróleo. Essa metodologia criada visou à confecção e apresentação de material paleontológico alternativo, dada a escassez de material paleontológico verídico para pesquisa-ensino.

O material utilizado para a elaboração dos modelos foi os descartes de isopor. Além de retirar esses resíduos de isopor inutilizados do meio ambiente, deu-se uma utilidade a esse detrito, um sentido simbólico para auxiliar na estruturação do conhecimento paleontológico, um sentido prático para o reaproveitamento de resíduos.

Diante do exposto, pode-se considerar que o conhecimento científico deste trabalho se construiu, principalmente, através do estudo, inovação e dedicação dos alunos, numa busca pelo fazer-aprender.

\section{REFERÊNCIAS BIBLIOGRÁFICAS}

1. ANELLI, L. E.; OYAKAWA, J.; FIGUEIREDO, S. S. C.; \& CALÇA, C. P. Moldagem e Replicação de Fósseis. In: CARVALHO, I. S. (Org.). Paleontologia. Vol. 2. Rio de Janeiro: Interciência, 2004.

2. BRITO, I. M. Geologia Histórica. Uberlândia: EDUFU, 2001.

3. CARVALHO, I. S. (Org.) Paleontologia. Vol. 1. Rio de Janeiro: Interciência, 20 04. Brasil. DECRETO-LEI № 4.146, de 04 de março de 1942. Dispõe sobre a proteção d os depósitos fossilíferos.

4. DULEBA, W.; PETRI, S.; \& COIMBRA, J. C. F. Foraminínferos, Tecamebas e Ostracodes SubRecentes e Fósseis do Quaternário do Brasil. In: Revista do Instituto de Geociências da USP. Publicação Especial. São Paulo: USP, 2007.

5. GUELERI, R. A.; GUILHERME, I. R.; \& SEMENSATTO JR, D. L. An Ontology to Support in the Identification of Foraminifera. In: Seminário de Pesquisa em Ontologia no Brasil, 11 e 12 de Agosto. São Paulo, 2008.

6. JERÔNIMO, V. L. Resíduos Sólidos. João Pessoa: CEFET-PB, 2004. 
7. MENDES, J. C. Paleontologia Geral. 2a . Ed. Rio de Janeiro: LTC, 1982.

8. MUNIZ, G. C. B. Novos Moluscos da Formação Gramame, Cretáceo Superior, dos Estados da Paraíba e de Pernambuco, Nordeste do Brasil. Recife: UFPE/DG, 1993.

9. REIS, M. Completamente Química. São Paulo: FTD, 2003.

10. SAMPAIO FILHO, H. A.; NUNES, J. L. GONÇALVES, P. M. Implantação do Salão de Fósseis "Bahia - 500 Mil Anos Antes de Cabral" - Museu Geológico da Bahia. In: Anais do XLI Congresso Brasileiro de Geologia A Geologia e o Homem, de 15 a 20 de Setembro de 2002. João Pessoa: SBG, 2002.

11. SIMONE, L. R. L. Fossil Mollusc of Brazil. São Paulo: Instituto Geológico: 1994.

12. TINOCO, I. M. Foraminíferos Quaternários de Olinda, Estado de Pernambuco. Rio de Janeiro: Serviço Gráfico do Instituto Brasileiro de Geografia e Estatística, 1958. (Monografia de Graduação). 